\section{Taskforce Tarmed SGUM/SSUM}

Der Bundesrat hat den Entwurf seines subsidiären Tarifeingriffes in Form einer Version 1.09 BR zur Vernehmlassung vorgestellt. Mit diesem sollen ab 1.1.2018 über 700 Millionen Franken eingespart werden. Leider ist der Entwurf nicht nur unausgewogen und auch möglicherweise sogar rechtlich nicht haltbar, sondern in vielen Inhalten kontraproduktiv. Auch viele Ultraschallleistungen werden zum Teil erheblich gekürzt. Zahlreiche Standesorganisationen haben denn auch ihre Antworten verfasst und zeitgerecht eingereicht. Weitere Einzelheiten sind auf www.arzttarif.ch ersichtlich.

Parallel dazu laufen aber die Arbeiten am Projekt TARCO der FMH unvermindert weiter. Dieses verspricht eine sachbezogene und betriebswirtschaftlich korrekte Aufarbeitung. Vor Mitte bis Ende 2018 dürfte allerdings die definitive Fassung kaum ausgearbeitet sein. Gut Ding will Weile haben!

Beat Dubs

Leiter Taskforce Tarmed 